\title{
Volatility Strategies for Global and Country Specific European Investors
}

\author{
Marie Brière ${ }^{1,3}$ \\ Amundi Asset Management, France. \\ Université Libre de Bruxelles, SBS-EM, Centre Emile Bernheim, Belgium. \\ Email : marie.briere@amundi.com \\ Jean-David Fermanian ${ }^{2,4}$ \\ Université de Paris-Dauphine, Ceremade, France. \\ Centre de Recherche en Économie et Statistiques (CREST), France. \\ Email : jean-david.fermanian@ensae.fr \\ Hassan Malongo ${ }^{2,3}$ \\ Amundi Asset Management, France. \\ Université de Paris-Dauphine, Ceremade, France. \\ Email : hassan.malongo@amundi.com

\section{Ombretta Signori ${ }^{5}$} \\ AXA Investments Managers, France. \\ Email : ombretta.signori@axa-im.com
}

This Version : 17 october, 2010

${ }^{1}$ Université Libre de Bruxelles, Centre Emile Bernheim - Solvay Business School, Av. F.D. Rooselvelt, 50, CP 145/1, 1050 Brussels, Belgium.

${ }^{2}$ Université de Paris-Dauphine, Centre de Recherche en Mathématiques de la Décision (Ceremade), Place du Maréchal De Lattre De Tassigny, 75775 Paris, France.

${ }^{3}$ Amundi Asset Management, 90 Boulevard Pasteur, 75015 Paris, France.

${ }^{4}$ Centre de Recherche en Économie et Statistiques (CREST), 15 Boulevard Gabriel Peri, 92245 Malakoff, France.

${ }^{5}$ AXA Investment Managers, 100 Esplanade du Général de Gaulle, 92932 Paris, France. 


\begin{abstract}
Adding volatility exposure to an equity portfolio offers interesting opportunities for long-term investors. This article discusses the advantages of adding a long volatility strategy for a protection to a global European equity portfolio and to specific equity portfolios based in "core" or "peripheral" countries within the euro zone. A European investor today has the choice of investing in US or European equity volatility. We check whether a long volatility strategy based on VSTOXX futures is better than a strategy based on VIX futures. The benefit of using volatility strategies as a hedge for equities is shown through a Mean/Modified-CVaR portfolio optimization. We find that long volatility strategies offer valuable protection to all European equity investors. A long volatility strategy based on VSTOXX futures offers better protection than a similar one based on VIX futures. It reduces the risk of an equity portfolio more significantly, while providing more attractive returns. For specific European investors, and despite major differences in local European equity markets, our long volatility strategy shows a certain homogeneity and provides efficient protection, whatever the country.
\end{abstract}

Keywords : Investment strategy, Portfolio choice, Conditional Value-at-Risk, Implied volatility, Hedging. JEL codes : G11, G15, G17 


\section{INTRODUCTION}

Since the financial crisis in 2008-2010, many investors have shown greater interest in volatility strategies because of their attractive characteristics. Volatility tends to spike in times of market stress, and its movements are known to be negatively correlated with risky asset returns. The US subprime crisis of 2008 or the recent European sovereign debt crisis of 2010 are particularly good examples of this phenomenon (see Figure 1 in Appendix II).

Accordingly, adding volatility exposure to an equity portfolio can offer interesting opportunities for long-term investors. This has been well documented in the academic literature. Daigler and Rossi (2006) show that the strong negative correlation between the S\&P 500 index and the VIX (implied volatility index of the S\&P 500) offers significant diversification benefits. Dash and Moran (2005) explore the relationship between the VIX and hedge fund returns. They show that being long implied volatility is an excellent hedge against the risks of portfolios that contain alternative-type investments. Brière et al. (2010) examine the advantage of incorporating two volatility strategies into an equity portfolio for long-term investors. They use a long volatility strategy with time varying exposure that protects against equity risk and a short variance swap strategy that reduces the overall cost of the long volatility strategy and which is partially hedged by the long volatility strategy in times of crises. Taken separately, each of the strategies shifts the efficient frontier significantly outward, but combining the two produces even better results.

One of the simplest ways to get exposure to volatility is to invest in implied volatility futures such as VIX or VSTOXX futures. VIX futures are based on the 30-day implied volatility deduced from S\&P 500 traded options and were introduced on the CBOE in March 2004. VSTOXX futures are the equivalent ${ }^{1}$ of VIX futures in Europe and are based on EuroStoxx 50 options. They were introduced on the Eurex exchange market in September $2005^{2}$.

1. VDAX, VCAC or VSMI are other implied volatility indices on respectively German, French and Swiss equity indices, but futures on these indices are not listed yet.

2. These futures are listed with monthly expiries which are usually on the Wednesday prior the second to last Friday of their respective expiration/maturity month. 
While the situation of US investors has been extensively investigated, this is not the case in Europe. At present, such an investor has the choice between two types of strategies : one based on VSTOXX futures that appears as the most logical hedge for his European equity risk but which is relatively less liquid than the US equivalent strategy based on VIX, or the alternative VIX futures strategy. As most of the work on this topic has been done for US investors in the academic literature, we reconsider the problem from the viewpoint of European investors facing this choice. We first analyze the relationship between a global European equity index and long volatility strategies based on VIX and VSTOXX futures. Next, we check whether these strategies provide a way to hedge European country equity risk exposures. Specific country equity markets are represented by a selection of core European countries (France and Germany) and peripheral countries (Portugal, Ireland, Greece and Spain) which are currently experiencing economic difficulties (high unemployment and budget deficits, high risk on their sovereign debt). The "country per country" analysis is interesting in itself, beside obvious practical implications. Indeed, it is a way of checking the robustness of a long volatility strategy as a hedge against equity exposures, when the strategy is related to the Euro (or US)-zone globally and the exposure is purely national. Our results show that it is not clear whether the choice between VSTOXX and VIX futures is definitive, independently of the specification of each national equity market. In fact, some disparities may exist across the European equity markets themselves. And even if the correlation between US and EU equity indices is high, indices in implied volatility like VSTOXX and VIX highlight the equity risks for two different regions which rely partly on different specific drivers. We finish the study by giving the benefit of using this kind of volatility strategy through a Mean/Modified-CVaR portfolio optimization for both the global and the specific European investor.

We believe this research is original for two reasons. First, unlike most previous research (e.g. Dash and Moran (2005) or Daigler and Rossi (2006)), we propose a long volatility strategy which uses real future prices data and thus takes explicitly into account the cost (or the gain) of rolling these futures positions. Actually, in the academic literature, most authors use the spot level of the implied volatility index directly instead of the futures prices and 
without calculating the rolling cost (or gain) associated with these futures when they evaluate their volatility strategies. In this article, we build a long volatility strategy which is more realistic and we examine the advantage of incorporating it into an equity portfolio. Second, since the returns of volatility strategies are asymmetric and leptokurtic, we propose an appropriate optimization technique through a measure of risk which captures higher order moments. In fact, most of the previous studies in the literature use mean-variance optimization even if there is empirical evidence that financial asset returns are not normally distributed (e.g. Andersen et al. (2000), Goetzmann et al. (2002)). Investors who are sensitive to extreme events of return distributions should not use mean-variance optimization because by ignoring the underlying skewness and kurtosis, this technique allocates the assets sub-optimally (Leland (1999), Cremers et al. (2005)). In this paper, our reference measure of risk is a Conditional "Modified" Value-at-Risk (CVaR) proposed by Cao et al. (2010). We use this semi-parametric measure of risk because it provides a good picture of tail risks, especially under strongly adverse conditions.

We find that long volatility strategies offer valuable protection to European equity investors. A long volatility strategy based on VSTOXX futures offers better protection than the addition of a long volatility strategy based on VIX futures for a global European investor. To be specific, a long volatility VSTOXX strategy offers a way of reducing more than half of the modified CVaR of the equity-only portfolio of a global European investor, and the returns are more attractive than when using the VIX strategy. This is explained by the fact that rolling VSTOXX futures is much less costly than rolling VIX futures, as the volatility term structure tends to be steeper for VIX than for VSTOXX futures. For all specific European investors, our volatility hedging strategy has shown a certain homogeneity. Our findings show that adding futures on implied volatility to a specific equity portfolio provides results close to those obtained with a global European equity portfolio, at least qualitatively. In particular, we find that specific equity portfolios with VSTOXX futures are generally better than those with VIX futures.

The paper is organized as follows. In section 2, we detail the methodology used to build the volatility strategy and we discuss the theoretical background of our measure of risk. In section 3 we present the data used and discuss 
the descriptive statistics. Section 4 is related to the empirical results of optimal portfolios for a global European investor and for specific country investors. Section 5 summarizes our conclusions and outlines the implications for further research.

\section{METHODOLOGY}

\section{Long exposure to volatility}

We consider a European equity investor willing to take systematic exposure to volatility in his equity portfolio. From a practical point of view, this investor can hedge his equity risk by using volatility options or rolling futures on implied volatility. As in Brière et al. (2010), we build a volatility strategy which tries to take advantage of the mean-reverting property of volatility and which rolls a long position in futures. Our long volatility strategy consists in rolling monthly the third ${ }^{3}$ nearest futures contract (compared to the last day of the current month). Broadly, the investment consists in putting a portion of the investor's capital in cash (which is used for the futures margin deposits) and another portion in a specified number of VSTOXX or VIX futures such that the impact of a 1-point variation in the prices of the futures is equal to $\frac{1}{F_{t-1}} * 100 \%$ (where $F_{t-1}$ is the price of the future at time t-1). As the three-month VIX and VSTOXX futures prices are available only since February 2007, we have estimated their prices before this date by estimating the average linear relation between futures prices and their respective index during their period of existence. These estimated values of this linear model provide the futures prices before their period of existence ${ }^{4}$. Moreover, when we constructed the volatility strategy, we took into account the cost (or the gain $)^{5}$ of rolling these futures. As we want to use the volatility strategy as an equity

3. Actually, several futures contract maturities are possible (one month, two months etc). In general, we seek a hedge against equity index movements in the short run through volatility exposure. Thus, the shorter the maturity of the futures, the better our hedge should perform. In fact, this is not the case. Indeed, rolling one-month or two-month futures at the end of each month generates negative returns for a similar level of risk. Conversely, three-month futures seem to provide a good balance between relevance (time-horizon of the underlying) and smoothness.

4. The $R^{2}$ of the linear regression between the three-month VIX futures and VIX index over the period from February 2007 to December 2010 is equal to 0.78 while it is equal to 0.75 for the regression with the three-month VSTOXX futures over the period from February 2007 to December 2010.

5. The cost (or gain) of rolling the three-month VIX and VSTOXX futures prior to their respective period of existence is taken equal to the average cost (or gain) to roll these futures during their period of existence. 
hedge and exploit the negative correlation between implied volatility and equity returns, we have normalized the volatility exposure by setting its monthly $95 \%$ modified CVaR at the same targeted level of risk as the equity reference asset class. Implicitly, it means that we want the worst scenarios for the index to be the best ones for the volatility strategy, broadly speaking. Once calibrated, we have written the return of the volatility strategy as the risk-free return plus a fixed proportion (also called degree of leverage) of the capital in VSTOXX or VIX futures (see Brière et al. (2010) for more details on the strategy). The returns of the long volatility strategies between $t$ and $t+1$ are then calculated as follows :

$$
\begin{aligned}
& r_{t+1}^{L V}=r_{t+1}^{f}+\theta * P \& L^{\text {Futures }} \\
& P \& L^{\text {Futures }}=\frac{1}{F_{t}}\left(F_{t+1}-F_{t}\right)
\end{aligned}
$$

where $r_{t}^{f}$ is the cash return and $F_{t}{ }^{6}$ is the price of the future at time $t . \theta$ is the so-called degree of leverage, chosen so that the modified CVaR of the long volatility strategy is the same as the modified CVaR of the equity portfolio. The detailed methodology used to calibrate $\theta$ is given in Appendix I.

\section{Risk measure}

When asset returns are not normally distributed, their variance is no longer an appropriate measure of risk. To be more specific, variance often leads to an underestimation or a biased view of the true underlying risks since asset returns are generally both skewed and leptokurtic. Even if there is empirical evidence that financial asset returns are not normally distributed, one of the most commonly used measures of risk in the financial industry is Value-at-Risk $^{7}$ (VaR) based on the standard normal distribution. Its success is largely due to its equivalence with the standard deviation in the case of underlying Gaussian distributions (see Jorion (2007) e.g.). As an alternative

\footnotetext{
6. The investor should consider that the contract value for VSTOXX futures is EUR 100 per index point of the underlying while it is $\$ 1000$ per index point of the underlying for VIX futures. As the amount to invest in the long volatility strategy evolves with time, the investor will invest each month in a number of futures equal to $\frac{N_{t} * \theta}{M * F_{t}}$ where $N_{t}$ is the part of the investor's capital allocated to the volatility strategy (cash + futures position) at date t and $M=100$ if he/she invests in VSTOXX futures or $M=1000$ if he/she invests in VIX futures.

7. VaR is defined as the maximum potential loss over a time of period given a specified probability $\alpha$.
} 
to dealing with non-normally distributed assets, Favre and Galeano (2002) have proposed a new measure of risk called "modified Value-at-Risk". This risk measure takes into account higher-order moments and replaces the quantile of the standard normal distribution with a modified quantile approximated by the Cornish-Fisher expansion ${ }^{8}$ (see e.g. Ord and Stuart (1999)). Modified VaR is defined by :

$$
\begin{gathered}
\text { Mod_VaR }(1-\alpha)=-[\mu+\sigma * Q(\alpha)] \\
Q(\alpha)=z_{\alpha}+\frac{1}{6}\left(z_{\alpha}^{2}-1\right) * S+\frac{1}{24}\left(z_{\alpha}^{3}-3 z_{\alpha}\right) *(K-3)-\frac{1}{36}\left(2 z_{\alpha}^{3}-5 z_{\alpha}\right) * S^{2},
\end{gathered}
$$

where $\mu, \sigma, \mathrm{S}$ and $\mathrm{K}$ denote the mean, the standard deviation, the skewness and the kurtosis of the portfolio's returns respectively. Obviously, $z_{\alpha}$ denotes the $\alpha$-quantile of the standard normal distribution $\mathcal{N}(0,1)$.

Another way to measure the risk of a portfolio is to invoke Conditional Value-at-Risk (CVaR), as introduced by Rockafellar and Uryasev (2000). It is defined as the average of the loss quantiles exceeding the VaR at the threshold $\alpha$, but rescaled by $1-\alpha$. In the case of continuous underlying distributions, as in our framework, CVaR is equal formally to the Expected Shortfall (also called Tail Conditional Expectation) ${ }^{9}$ : see Tasche (2002). Since CVaR is a so-called "coherent measure of risk", in the sense of Artzner et al. (1999), it has become a standard in risk management. In this article, however, our reference measure of risk will be Conditional Value-at-Risk (CVaR) based on a "modified Value-at-Risk". This measure is a semi-parametric approach proposed by Cao et al. (2010), who introduce it to hedge different long positions in equity indices with index futures contracts. We have chosen modified CVaR instead of modified VaR because the latter risk measure still suffers from some well-known "drawbacks" of a VaR measure. Most of these are well documented in the literature (see Danielsson (2002), e.g.). For instance, instability is greater for VaR (modified or not) than for CVaR (modified or not) when dealing with very high/small levels of $\alpha$, and particularly when the return distributions are strongly non normal (Boudt et

8. The higher-order moments taken into account in the modified VaR formula are skewness and kurtosis. Some authors have considered more "correction" terms in the Cornish-Fisher expansions and have found that increasing the order does not necessarily improve the approximation (see e.g. Jaschke (2002)).

9. The Expected Shortfall is defined as the average of the losses exceeding the VaR at the threshold $\alpha$. 
al. (2007)). Moreover, beside its property of coherence, CVaR is superior to VaR in optimization applications (Rockafellar and Uryasev (2000)) and has nicer mathematical properties ${ }^{10}$ than VaR. In practice, VaR is less than CVaR with the same confidence level, and a risk-averse investor may prefer CVaR because it copes with large losses when they occur. From an analytic point of view, the modified CVaR is written as :

$$
\text { Mod_CVaR }(1-\alpha)=\frac{1}{\alpha} \int_{1-\alpha}^{1} M o d_{-} V a R(x) d x=-\left[\mu+\frac{\sigma}{\alpha} \int_{1-\alpha}^{1} Q(1-x) d x\right] .
$$

Being simple to implement, this expression can be rewritten (Cao et al. (2010)) as

$$
\text { Mod_CVaR }(1-\alpha)=-\mu-\sigma *\left[M_{1}+\frac{1}{6}\left(M_{2}-1\right) * S+\frac{1}{24}\left(M_{3}-3 M_{1}\right) * K-\frac{1}{36}\left(2 M_{3}-5 M_{1}\right) * S^{2}\right]
$$

where $M_{i}=\frac{1}{\alpha} \int_{-\infty}^{z_{\alpha}} x^{i} f(x) d x$ and where $f($.$) is the standard normal probability density function.$

\section{THE DATA}

The study covers the period between January 1999 and December 2010. We use the MSCI EMU index in total return terms (performance includes reinvested dividend gains) for the global European equity index ${ }^{11}$. For the specific European equity indices, we rely on the MSCI France, Germany, Portugal, Ireland, Greece and Spain, also in total return ${ }^{12}$. To build the long volatility strategy, our underlyings are the third next to expire VSTOXX and VIX futures contracts (three-month maturity at buying date). The risk-free rate for cash investment is the one-month Euribor rate. As all the strategies are considered from the point of view of a European investor, we have converted the US investment in VIX futures into euro. VSTOXX and VIX futures are provided by Bloomberg, all other series (equity and implied volatility indices, EUR/USD exchange rate and Euribor rate) come from

\footnotetext{
10. In financial applications, the CVaR of a portfolio is a continuous and convex function of the underlying portfolio positions whereas its VaR may be a discontinuous function (Pflug (2000)).

11. As of December 2010, the MSCI EMU contains 267 stocks which represent 11 country indices within the European Monetary Union at December 31, 2010 : Austria (1.06\%), Belgium (3.04\%), Finland (3.42\%), France (32.71\%), Germany (28.35\%), Greece $(0.75 \%)$, Ireland $(0.82 \%)$, Italy $(9.27 \%)$, the Netherlands $(8.40 \%)$, Portugal $(0.88 \%)$, and Spain (11.30\%). Full details are available at www.msci.com.

12. MSCI France, Germany, Portugal, Ireland, Greece and Spain contain respectively 75, 52, 8, 4, 8 and 26 stocks in December 2010 .
} 
Datastream. Monthly closing prices are used for the equity indices, the VSTOXX and VIX indices, the risk-free rates, the exchange rates and the corresponding monthly settlement prices of the futures contracts.

Table 1 (Appendix III) provides descriptive statistics for the MSCI-EMU index and the two long volatility strategies over the period February 1999 and December 2010. In terms of annualized returns, the long volatility strategy based on VSTOXX futures (LV-VSTOXX) appears to be the most attractive, followed by the long volatility strategy based on VIX futures (LV-VIX) and the equity index (4.50\%, $2.84 \%$ and $1.76 \%$ respectively). The substantial difference in performances between the long volatility strategies based on VSTOXX and VIX futures is explained by the fact that the former are less costly to roll than the latter ${ }^{13}$. To keep a volatility exposure month after month, the investor has to buy at month end the third to expire future and sell the one bought the month before (which now becomes the second to expire), thus paying (or receiving) each month the spread between the third and the second futures contracts. This induces a cost (or a gain) which can be substantial : the rolling gain is $0.43 \%$ for VSTOXX futures, whereas it is costly $(-1.16 \%)$ for VIX futures ${ }^{14}$. This explanation is highlighted by Figures 2 and 3 (Appendix II). The spread between the third and the second futures VIX contracts is $61.7 \%$ of the time larger than the equivalent VSTOXX spread (Figure 2). Even if this spread decreases with maturity (see Figure 3), it appears that it is always larger for VIX than for VSTOXX futures.

Modified CVaR has been calibrated ${ }^{15}$ to be identical for the volatility strategies and the equity exposure. However, volatility differs quite sharply. Whereas it is $19.45 \%$ for equities, it is much stronger for both volatility strategies (33.89\% and $30.76 \%$ for VSTOXX and VIX respectively). An analysis of higher-order moments shows that the three assets' distribution departs strongly from normality. While the skewness of the MSCI EMU is negative (-0.38), confirming the high extreme risks of the equity exposure, it is strongly positive for both vola-

\footnotetext{
13. Note that the observed differences in performance between the two strategies are not due to exchange rates. We obtained very similar results when we considered the strategies' performances in local currency terms.

14. If we did not take into account this rolling cost, the annualized return of the long volatility strategies would be much closer, $4.07 \%$ for VSTOXX vs $4.00 \%$ for VIX futures respectively.

15. VSTOXX and VIX strategies have been calibrated with a parameter $\theta$ respectively equal to 0.63 and 0.70 .
} 
tility strategies (1.39 and 1.66 for VSTOXX and VIX respectively). This confirms the attractive role that long volatility strategies may play in an equity portfolio, providing valuable protection against the leftward asymmetry of the equity exposure. The kurtosis of all three assets is greater than $3(4.00,7.27$ and 10.60 for the equity index, VSTOXX and VIX respectively) and the Jarque-Bera statistic rejects the null hypothesis of normality for the three series at the $5 \%$ significance level. These results confirm the importance of taking into account the higher-order moments when evaluating the risk.

Table 2 (Appendix III) provides the correlation matrix between the European equity index and the two volatility strategies. We observe that the two volatility strategies are strongly correlated $(0.80)$. The correlation coefficient between European equities and volatility strategies is -0.66 for VSTOXX while it is equal to -0.63 for the VIX. This strong negative correlation confirms the role of volatility strategies as a partial hedge for the equity exposure (Dash and Moran (2005), Daigler and Rossi (2006), Brière et al. (2010)). However, even if VSTOXX futures appear to have a slightly lower correlation with equities than do VIX futures, this difference is not significant when we apply the Hotelling/Williams test ${ }^{16}$.

Table 3 (Appendix III) provides descriptive statistics for the equity indices of specific countries over the period February 1999 to December 2010. The Spanish equity index has the highest annualized returns (4.08\%) followed by France and Germany $(2.48 \%$ and $2.40 \%$ respectively). Ireland and Greece have the worst returns $(-9.97 \%$ and $-7.16 \%$ respectively) and also the strongest volatility (23.10\% and $29.33 \%$ respectively) and modified CVaR ( $16.79 \%$ and $19.85 \%$ respectively) compared to the others indices (for example $13.77 \%$ for Portugal modified CVaR and $12.21 \%$ for France). These results are hardly surprising considering the recent European crisis, which has fuelled investors' concerns about the economic situation of these two countries (budget deficit, sovereign debt risk). Maximum drawdowns are also particularly high for both countries (-81.56\% for Ireland and $-76.43 \%$ for Greece). The analysis of higher order moments of the return distribution clearly highlights that all country returns

\footnotetext{
16. The Hotelling/Williams test checks whether the correlation between $\mathrm{Z}$ and $\mathrm{X}$ differs from the correlation between $\mathrm{Z}$ and $\mathrm{Y}$, where $\mathrm{X}, \mathrm{Y}$ and $\mathrm{Z}$ are three variables measured on the same set of observational units.
} 
are non normally distributed (negative skewness and excess kurtosis), a result confirmed by the Jarque-Bera test.

Table 4 (Appendix III) reports the correlation matrix between country equity returns and the two volatility strategies. As expected, we observe a stronger correlation of stock indices between the "core" European countries, namely France and Germany (0.88), than between the peripheral ones (around 0.5, for Portugal, Ireland, Greece and Spain). The correlations between the country equity indices and the long volatility strategies based on VSTOXX and VIX futures are strongly negative (between -0.45 and -0.66). These negative correlations are greater with the core European countries than with the peripheral ones. Moreover, they are always higher with VSTOXX futures than with VIX futures. In line with what we found for global European equity investment, we observe that VSTOXX futures provide a slightly higher diversification benefit than VIX futures for each specific European country investor, but the differences in correlations are not significant when the Hotelling/Williams test is applied.

\section{OPTIMAL PORTFOLIOS}

In what follows, we examine the advantages for a European investor of adding a volatility strategy based on VSTOXX or VIX futures. We first build optimal portfolios made of global European equities and volatility strategies and then compare them with a pure equity investment. Next, we consider the case of specific European investors who want to hedge their local equity exposure with volatility strategies. We check if these advantages are unique whatever the specific characteristics of each equity market.

\section{Optimal portfolio for a global European Investor}

To determine the interest of adding a systematic exposure to volatility to a European equity portfolio and compare VIX with VSTOXX futures investments, we consider two basic types of portfolios : Equity with a long volatility strategy based on VSTOXX futures and Equity with a long volatility strategy based on VIX futures, and 
we compare them with the global European equity investment. Efficient frontiers made of equity-VIX and equityVSTOXX (Figure 4 in Appendix II) are calculated by minimizing the Modified CVaR at threshold $\alpha=95 \%$ for different levels of expected returns. We provide global minimum CVaR portfolios compositions and performances in Table 5 (Appendix III).

Allocating around $45 \%$ (45.47\% for the VSTOXX strategy and $42.77 \%$ for the VIX) of the portfolio to the long volatility strategies is necessary to achieve the minimum level of risk. The addition of long volatility strategies makes it possible to reduce more than half the modified CVaR of the equity-only portfolio (4.97\% for the equity-VSTOXX and $5.16 \%$ for the equity-VIX vs $13.14 \%$ for the equity-only portfolio). Moreover, both portfolios have much more attractive returns $(5.94 \%$ vs $4.72 \%)$ than the equity-only portfolio $(1.76 \%)$.

An analysis of extreme negative events shows that the maximum monthly loss is more than halved $(-5.85 \%$ and $-8.61 \%$ ) when including VIX and VSTOXX strategies respectively, compared with the equity-only portfolio (-17.53\%). Furthermore, looking at the maximum drawdown, we observe that the equity-VSTOXX portfolio has the smallest drawdown (-11.19\%) compared to the equity-VIX portfolio (-25.72\%) and the equity-only (-55.69\%). The portfolio's skewness also becomes positive (1.30 and 0.67 when adding VSTOXX and VIX respectively), and should be compared to the strong negative skewness of the equity-only investment (-0.38).

On the whole, our findings show that while both volatility strategies are attractive for hedging a strategic equity exposure, a long volatility strategy based on VSTOXX futures offers better protection for a European equity investor than a similar strategy based on VIX futures. Not only are extreme risks smaller, but the returns of the portfolio are also substantially higher. This is true not only for the minimum $95 \%$ modified CVaR but also for most levels of risk ${ }^{17}$. This result is highlighted by Figure 4 (Appendix II), which gives the efficient frontiers for the MSCI-EMU index with long volatility strategies. We clearly observe that the efficient frontier of the equity

17. We also optimized the portfolios with $\alpha=99 \%$ and we found that the results were robust. 
portfolio with VSTOXX futures is always above the efficient frontier of the equity portfolio with VIX futures and we see that this gap increases with the risk level of the strategy.

\section{Optimal portfolios for specific European investors}

Tables 6 and 7 (Appendix III) give the optimal weights and performances of the portfolios with VSTOXX and VIX futures (respectively) for the six specific country investors. By and large, for all country investors, our findings are consistent with what we found in the previous section. Adding around $45 \%$ (between $36.40 \%$ and 46.14\%) of a volatility strategy based on VSTOXX or VIX futures to a European equity portfolio greatly improves the risk-adjusted returns of the portfolios. The equity-VSTOXX portfolios have much higher annualized returns than the equity-VIX portfolios for all country investors. In some cases (Greece and Ireland), adding a VSTOXX strategy makes the portfolio's performances positive ( $1.07 \%$ and $0.01 \%$ respectively), whereas the inclusion of VIX strategies is not sufficient to counterbalance the strong negative performances of these equity markets $(-7.16 \%$ and $-9.97 \%$ respectively over the period).

As in the case of the global European investor, we observe that adding volatility exposure strongly reduces the risks of the equity-only portfolios. For example, the $95 \%$ modified CVaR of the German equity portfolio is equal to $16.06 \%$ (see Table 3, Appendix III) compared with $6.17 \%$ and $6.51 \%$ when we add long volatility based on VSTOXX and VIX futures respectively. Moreover, for all specific European investors, the addition of a long volatility strategy to an equity portfolio is an interesting way to reduce the maximum losses and maximum drawdown, without significantly diminishing the maximum monthly gain. For instance, for an Irish equity investor, the maximum drawdown $(-81.56 \%)$ is reduced to $-45.54 \%$ and $-40.46 \%$ when a volatility strategy with VIX and VSTOXX (respectively) is added, whereas the maximum gain is actually increased from $14.15 \%$ to $21.80 \%$. Finally, our results show that, on the whole, a long volatility investment in VSTOXX futures is a better hedge than VIX futures for all specific investors. Not only are returns significantly higher, but the portfolios also exhibit smaller modified CVaR and maximum drawdowns as well as higher positive skewness. 
Our hedging strategy with volatility futures has shown a remarkable homogeneity even though there may be disparities between European equity markets. Actually, country equity indices differ in terms of sector composition (Cooper and Kaplanis (1994), Tesar and Werner (1995)) and as economic difficulties are regional in nature, national markets respond differently to global shocks (Rouwenhorst (1998)). These disparities are reflected in the fact that European equity markets are not particularly highly correlated ${ }^{18}$. On the whole, for both the core EU economies (France and Germany) and the peripheral countries (Portugal, Ireland, Greece and Spain), we find that a long volatility strategy based on VSTOXX futures provides a good way to hedge local equity exposures. In fact, it is better than a similar VIX futures exposure. These results confirm that, compared with VIX, VSTOXX better reflects the financial risks ${ }^{19}$ in Europe.

\section{CONCLUSION}

Since the recent financial crisis, the need to find strategies for hedging exposures to risky assets, especially equities, has aroused a great deal of interest among long-term investors, and the appeal of volatility strategies has increased considerably. If the case of US investors has been extensively studied, the particular situation of European investors has not received much attention. This paper tries to fill the gap by investigating the interest of adding volatility exposure to a European equity portfolio, whether global or on a specific country index. In the context of the recent European crisis, we believe this research may be of practical value for many investors.

By and large, our results confirm the interest of adding volatility exposure (based on VSTOXX or VIX futures)

to a European equity portfolio. We show that a long volatility strategy based on VSTOXX futures offers better

18. Only Germany, France and Spain show a high correlation over the studied period (see Table 4, Appendix III).

19. For instance, when Fitch rating agency downgraded Greece's government debt to BBB+ from A- with a negative outlook at December 8, 2009, the EuroStoxx 50 index fell of $-2.70 \%$ whereas VSTOXX and VIX index increased of $13.81 \%$ and $2.53 \%$ respectively between December 7 and December 9, 2009. The impact of this news reflected by the spike of VSTOXX index, had affected more the European market than the US market. 
protection than a long volatility strategy based on VIX futures when dealing with a European equity investment, whether global or specific to a euro zone country. This result is explained by the fact that the steepness of the curve for VIX futures incurs a higher cost of carry compared with that for VSTOXX futures. In addition, as VSTOXX and VIX index price the risk in different regions with specifics drivers, the fall in the European equity index will be higher than that in the US equity index during a European crisis for instance. VSTOXX futures are then better candidates than VIX futures to hedge the European equity risk.

One of the limitations of our work relates to the period of time needed to roll the futures. Although the period from 1999 to 2010 contains some major economic and financial crises, we cannot be sure that, in future, there will be no volatility movements that induce high rolling costs and could impair the performances of the proposed hedge. Having a systematic long exposure in futures on implied volatility is still a sophisticated investment strategy that can produce substantial losses if not managed appropriately, according to the phases of the stock market. One interesting continuation of this work would be to build a tactical strategy based on specific signals and making it possible to refine the volatility exposure and buy an optimal number of futures on implied volatility, especially during periods of equity market stress. 


\section{References}

[1] Andersen J.V., Simonetti P. and Sornette D. (2000). Portfolio Theory for Fat Tails. International Journal of Theoretical and Applied Finance 3(3):523-535.

[2] Artzner P., Delbaen F., Eber J.M. and Heath D. (1999). Coherent Measures of Risk. Mathematical Finance $9(3): 203-228$.

[3] Boudt K., Croux C. and Peterson B.G. (2007). Estimation and decomposition of downside risk for portfolios with non-normal returns. MPRA Paper. University Library of Munich.

[4] Brière M., Burgues A. and Signori O. (2010). Volatility Exposure for Strategic Asset Allocation. The Journal of Portfolio Management 36(3):105-116.

[5] Cao Z., Harris R.D.F. and Shen J. (2010). Hedging and Value at Risk: A Semi-Parametric Approach. Journal of Futures Markets 30(8):780-794.

[6] Cooper I. and Kaplanis E. (1994). Home Bias in Equity Portfolios, Inflation Hedging, and Intemational Capital Market Equilibrium. Review of Financial Studies 7(1):45-60.

[7] Cremers J.H., Kritzman M. and Page S. (2005). Optimal Hedge Fund Allocations. The Journal of Portfolio Management 31(3):70-81.

[8] Daigler R.T. and Rossi L. (2006). A Portfolio of Stocks and Volatility. The Journal of Investing 15(2):99-106.

[9] Danielsson J. (2002). The emperor has no clothes: Limits to risk modelling. Journal of Banking \& Finance 26(7):1273-1296.

[10] Dash S. and Moran M.T. (2005). VIX as a Companion for Hedge Fund Portfolios. The Journal of Alternative Investments 8(3):75-80.

[11] Favre L. and Galeano J.A, (2002). Mean-modified Value at Risk Optimization With Hedge Funds. The Journal of Alternative Investment 5(2):21-25.

[12] Goetzmann W., Ingersoll J., Spiegel M. and Welch I. (2002). Sharpening Sharpe Ratios. NBER Working Paper No. 9116.

[13] Jaschke S.R. (2002). The Cornish-Fisher expansion in the context of deltagamma- normal approximations. Journal of Risk 4(4):33-52.

[14] Jorion P. (2007). Value At Risk. 3rd Ed., McGraw Hill.

[15] Leland H., (1999). Beyond mean-variance: risk and performance measures for portfolios with nonsymetric distributions. Working paper. Haas School of Business, U.C. Berkeley. 
[16] Ord K. and Stuart A. (1999). Kendall's Advanced Theory of Statistics, Volume 1 : Distribution Theory, 6th edition. Oxford University Press.

[17] Pflug G.C. (2000). Some remarks on the value-at-risk and the conditional value-at-risk, in Uryasev S.P. (ed), Probabilistic Constrainted Optimization: Methodology and Applications. Kluwer, Norwell, MA, 278-287.

[18] Rockafellar R.T. and Uryasev S.P. (2000). Optimization of conditional value-at-risk. Journal of Risk 2(3):2142.

[19] Rouwenhorst K.G. (1999). European Equity Markets and EMU: Are the Differences Between Countries Slowly Disappearing? Financial Analysts Journal 55(3):57-64.

[20] Tasche D. (2002). Expected Shortfall and Beyond. Journal of Banking \& Finance 26(7):1519-1533.

[21] Tesar L.L. and Werner I.M. (1995). Home Bias and High Turnover. Journal of International Money and Finance 14(4):467-492. 


\section{Appendices}

\section{Appendix I}

From a practical standpoint, we specify here the way the parameter $\theta$ is calibrated, as well as the optimization procedure we used to obtain the optimal portfolios. Let us denote

$-r_{t}^{f}$ the (monthly) short rate,

- $F_{t}$ the spot level of the future,

- $I_{t}$ the spot level of the equity index,

- $P_{t}^{L V}$ the $t$-value of the LV strategy,

- $P_{t}^{G}$ the $t$-value of the global strategy, mixing a certain amount of the index and of the LV strategy,

- $P_{t}^{F}$ is the market value of a future at $t$,

- $N_{t}^{L V}$ the amount of cash that will be invested in the LV strategy at $t$,

- $M$ the reference notional of a VIX (or VSTOXX) future,

- $m_{t}$ the number of futures contracts the investor will buy at $t$ and sell at $t+1$.

At $t=0, N_{0}^{L V}$ are invested in cash and the investor has entered into $m_{0}$ futures. We have then

$$
P_{0}^{L V}=N_{0}^{L V}=N_{0}^{L V} * 1+m_{0} * P_{0}^{F} \text { with } P_{0}^{F}=0 .
$$

At $t=1$, we have

$$
P_{1}^{L V}=N_{0}^{L V}\left(1+r_{1}^{f}\right)+m_{0} P_{1}^{F}=N_{0}^{L V}\left(1+r_{1}^{f}\right)+m_{0}\left(F_{1}-F_{0}\right) M .
$$

The P\&L between $t=0$ and $t=1$ is then

$$
\Delta P_{0}^{L V}=P_{1}^{L V}-P_{0}^{L V}=N_{0}^{L V} r_{1}^{f}+m_{0}\left(F_{1}-F_{0}\right) M .
$$

The associated return is

$$
\Delta P_{0}^{L V} / P_{0}^{L V}=r_{1}^{f}+m_{0}\left(F_{1}-F_{0}\right) M / N_{0}^{L V}
$$

Similarly, the P\&L generated between $t$ and $t+1$ is

$$
\Delta P_{t}^{L V}=P_{t+1}^{L V}-P_{t}^{L V}=N_{t}^{L V} r_{t+1}^{f}+m_{t}\left(F_{t+1}-F_{t}\right) M
$$

where $N_{t}^{L V}$ is the cash amount that is available at $t$. Here, since we sell the whole portfolio at the end of every month, $P_{t}^{L V}=N_{t}^{L V}$ simply. The associated return is then 


$$
\Delta P_{t}^{L V} / P_{t}^{L V}=r_{t+1}^{f}+m_{t}\left(F_{t+1}-F_{t}\right) M / N_{t}^{L V}
$$

The return of the long volatility strategy between $t$ and $t+1$ is given by

$$
\begin{gathered}
r_{t+1}^{L V}=\Delta P_{t}^{L V} / P_{t}^{L V}=r_{t+1}^{f}+\nu_{t} \cdot \Delta F_{t} / F_{t}, \\
\nu_{t}:=F_{t} m_{t} M / N_{t}^{L V} .
\end{gathered}
$$

We make the assumption that $\nu_{t}$ will be a constant $\theta$. As we want the level of risk of the long volatility strategy to equal the level of risk of the equity only portfolio, we calibrate $\theta$ by the equation

$$
\operatorname{Mod} \_C V a R_{\alpha}\left(\Delta P_{t}^{L V} / P_{t}^{L V}\right)=\operatorname{Mod} C V V a R_{\alpha}\left(\Delta I_{t} / I_{t}\right) .
$$

Thus, we get the estimate $\hat{\theta}$. The constancy of $\theta$ means that $m_{t} \simeq C s t / F_{t}$. Thus, the number of futures we buy is inversely proportional to the spot level of the future. This pattern of the strategy makes it possible to profit from the mean reverting properties of volatility : the investor's exposure is increased when volatility is low, it is decreased when volatility is high (Brière et al. (2010)). Moreover, before building the optimized portfolios, we have assumed that the index strategy is static in the sense that we do not use cash regularly to buy/sell additional amounts of the equity index. Our optimization procedure consists in finding a parameter $\beta \in[0,1]$ that is the percentage of cash which will be invested in the equity index at the initial date. Such a parameter $\beta$ minimizes the $95 \%$ modified CVaR of the global strategy. Our optimization problem is then

$$
\hat{\beta}=\arg \min _{\beta} C V a R_{\alpha}\left(\Delta P_{t}^{T} / P_{t}^{T}\right) .
$$

With the same methodology as above, the returns of the global strategy between $t$ and $t+1$ are now

$$
\Delta P_{t}^{G} / P_{t}^{G}=\beta \cdot \Delta I_{t} / I_{t}+(1-\beta) \cdot \Delta P_{t}^{L V} / P_{t}^{L V} .
$$

The calibrated values of $\beta$ are shown at the bottom of Tables 5-7. 


\section{Appendix II}

FIgURE 1 - Daily closing prices on the EuroStoxx 50, VSTOXX and VIX index

This figure displays monthly closing prices on the EuroStoxx 50, VSTOXX and VIX index over the period February 1999-December 2010.

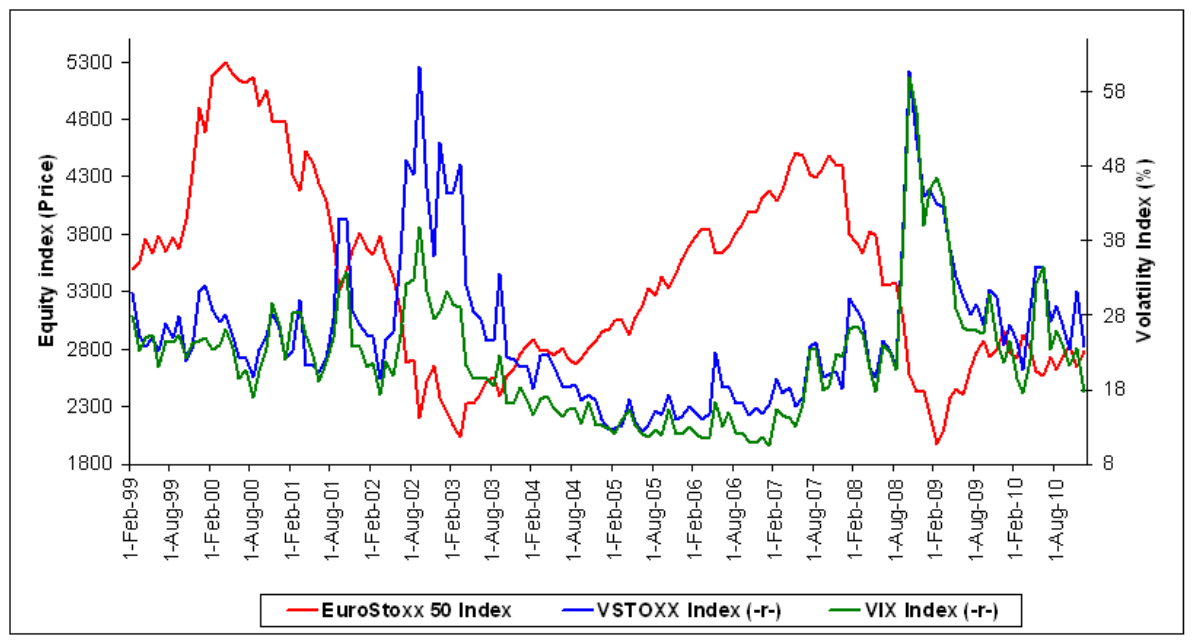

Figure 2 - Spread between the third and the second month futures

This figure displays the monthly spread (in \% volatility) between the third and the second futures prices of VSTOXX and VIX over the period February 2007-December 2010.

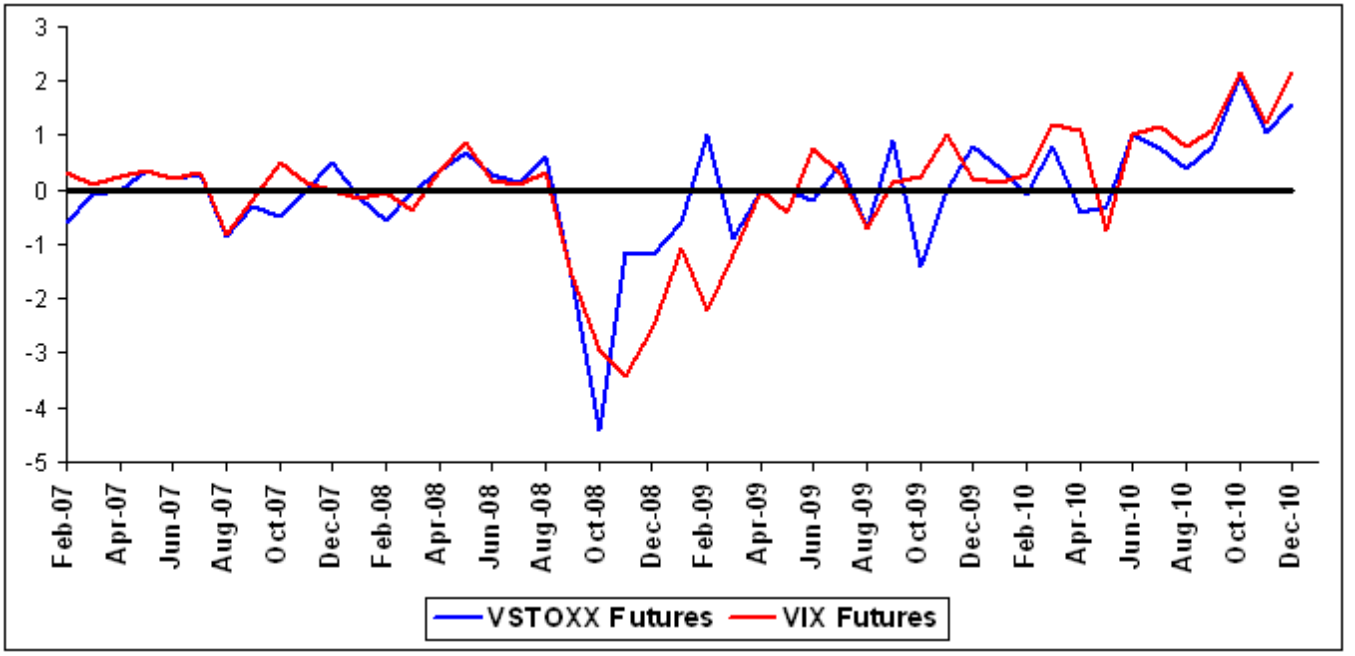


Figure 3 - Average volatility term structure

This figure displays the average price of the first, second and third futures contracts for VSTOXX and VIX over the period February 2007-December 2010.

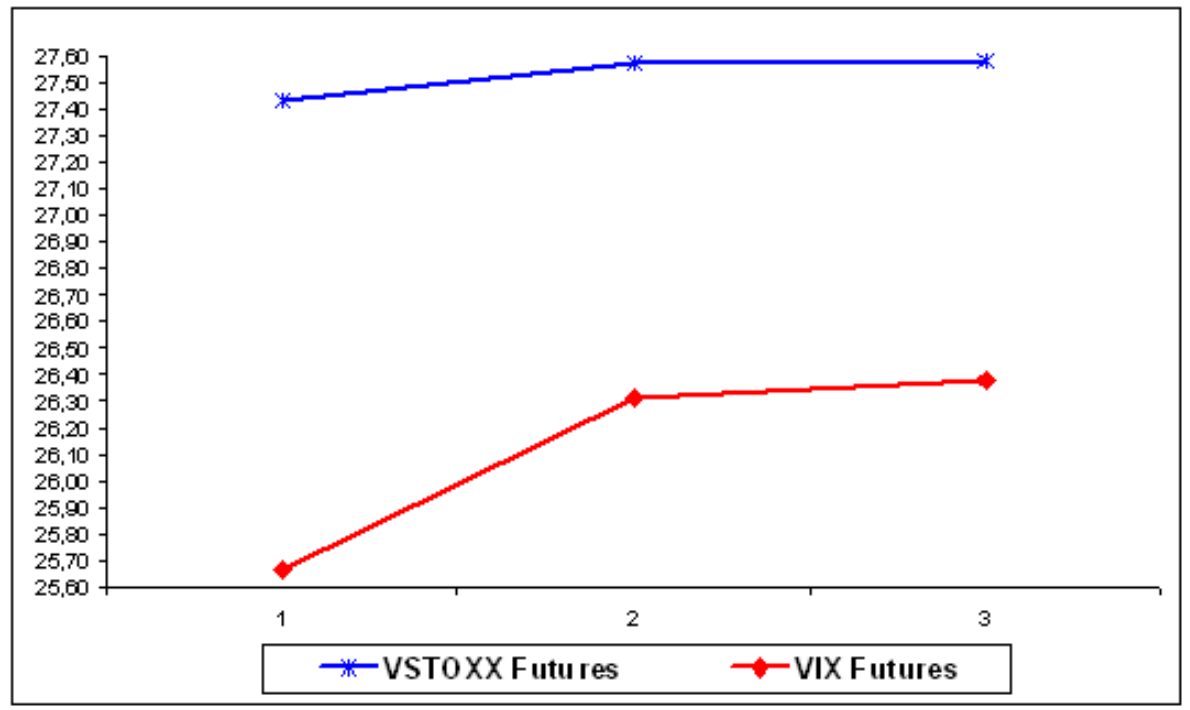

FiguRE 4 - Efficient Frontiers for global European investor This figure displays the efficient frontiers of MSCI-EMU with VSTOXX (1) and MSCI-EMU with VIX (2) over the period February 1999-December 2010.

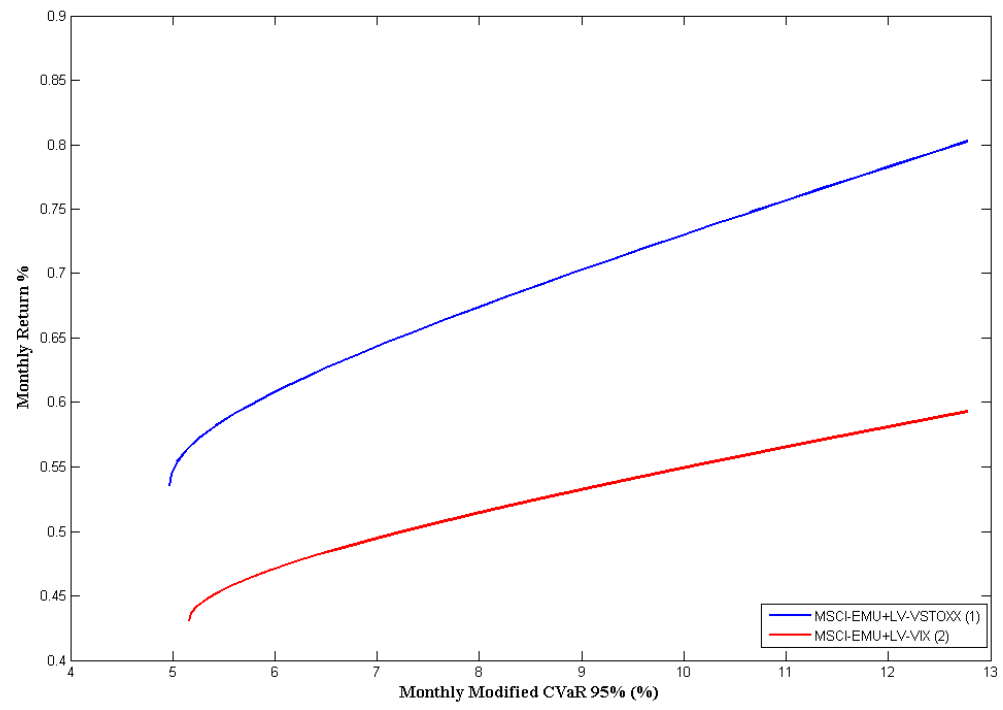




\section{Appendix III}

TABLE 1 - Descriptive Statistics for MSCI EMU index and long volatility strategies

This table reports the descriptive statistics for the MSCI EMU index and the long volatility strategies based on VSTOXX and VIX futures over the period February 1999-December 2010.

\begin{tabular}{c|c|c|c}
\hline & MSCI-EMU & LV-VSTOXX & LV-VIX \\
\hline Ann. Geo. Mean & $\mathbf{1 . 7 6 \%}$ & $\mathbf{4 . 5 0 \%}$ & $\mathbf{2 . 8 4 \%}$ \\
Ann. Std. Dev. & $19.45 \%$ & $33.89 \%$ & $30.76 \%$ \\
Max Monthly Loss & $-17.53 \%$ & $-19.68 \%$ & $-16.98 \%$ \\
Max Monthly Gain & $16.18 \%$ & $48.26 \%$ & $53.67 \%$ \\
Max Drawdown* & $-55.69 \%$ & $-47.98 \%$ & $-49.79 \%$ \\
Skewness & -0.38 & 1.39 & 1.66 \\
Kurtosis & 4.00 & 7.27 & 10.60 \\
Mod. CVaR (95\%)** & $\mathbf{1 3 . 1 4 \%}$ & $\mathbf{1 3 . 1 4 \%}$ & $\mathbf{1 3 . 1 4 \%}$ \\
Jarque-Bera Stat (95\%)*** & 9.31 & 154.50 & 410.21 \\
\hline
\end{tabular}

* The maximum drawdown is defined as the largest drop of a given asset within the sample period.

** VSTOXX and VIX strategies have been calibrated with a parameter $\theta$ respectively equal to 0.63 and 0.70 . *** The Jarque-Bera statistic is $\chi^{2}(2)$ distributed under the null hypothesis of normality of residuals.

\section{TABLE 2 - Correlation matrix between MSCI EMU index and long volatility strategies}

This table gives the correlation matrix between the MSCI EMU index and the long volatility strategies based on VSTOXX and VIX futures over the period February 1999-December 2010.

\begin{tabular}{c|ccc}
\hline & MSCI-EMU & LV-VSTOXX & LV-VIX \\
\hline MSCI-EMU & & & \\
LV-VSTOXX & -0.66 & & \\
LV-VIX & -0.63 & 0.80 & \\
\hline
\end{tabular}


TABLE 3 - Descriptive statistics for specific countries equity indices

This table gives the descriptive statistics of specific country equity indices over the period February 1999-December 2010 .

\begin{tabular}{c|c|c|c|c|c|c}
\hline & MSCI France & MSCI Germany & MSCI Portugal & MSCI Ireland & MSCI Greece & MSCI Spain \\
\hline Ann. Geo. Mean & $\mathbf{2 . 4 8 \%}$ & $\mathbf{2 . 4 0 \%}$ & $\mathbf{- 0 . 6 2 \%}$ & $\mathbf{- 9 . 9 7 \%}$ & $\mathbf{- 7 . 1 6 \%}$ & $\mathbf{4 . 0 8 \%}$ \\
Ann. Std. Dev. & $18.71 \%$ & $23.09 \%$ & $18.89 \%$ & $23.10 \%$ & $29.33 \%$ \\
Max Monthly Loss & $-15.98 \%$ & $-24.93 \%$ & $-19.97 \%$ & $-22.49 \%$ & $-29.88 \%$ \\
Max Monthly Gain & $13.55 \%$ & $20.94 \%$ & $13.37 \%$ & $14.15 \%$ & $24.90 \%$ & $-17.22 \%$ \\
Max Drawdown* & $-58.14 \%$ & $-67.91 \%$ & $-57.03 \%$ & $-81.56 \%$ & $-76.42 \%$ & $-49.58 \%$ \\
Skewness & -0.38 & -0.35 & -0.56 & -0.73 & -0.21 & -0.31 \\
Kurtosis & 3.54 & 4.61 & 4.60 & 3.50 & 3.92 \\
Mod. CVaR (95\%) & $\mathbf{1 2 . 2 1 \%}$ & $\mathbf{1 6 . 0 6 \%}$ & $\mathbf{1 3 . 7 7 \%}$ & $\mathbf{1 6 . 7 9 \%}$ & $\mathbf{1 9 . 8 5 \%}$ & $\mathbf{1 3 . 9 6 \%}$ \\
Jarque-Bera Stat(95\%)** & 5.11 & 18.36 & 22.78 & 14.09 & 6.13 & 7.29 \\
\hline
\end{tabular}

* The maximum drawdown is defined as the largest drop of a given asset within the sample period.

** The Jarque-Bera statistic is $\chi^{2}(2)$ distributed under the null hypothesis of normality of residuals.

TABLE 4 - Correlation matrix between Country equity indices and long volatility strategies This table reports the correlation matrix between the country equity indices and the long volatility strategies based on VSTOXX and VIX futures over the period February 1999-December 2010.

\begin{tabular}{|c|c|c|c|c|c|c|}
\hline & MSCI France & MSCI Germany & MSCI Portugal & MSCI Ireland & MSCI Greece & MSCI Spain \\
\hline MSCI France & & & & & & \\
\hline MSCI Germany & 0.88 & & & & & \\
\hline MSCI Portugal & 0.67 & 0.60 & & & & \\
\hline MSCI Ireland & 0.60 & 0.52 & 0.49 & & & \\
\hline MSCI Greece & 0.46 & 0.41 & 0.43 & 0.41 & & \\
\hline MSCI Spain & 0.86 & 0.78 & 0.68 & 0.56 & 0.46 & \\
\hline LV-VSTOXX & -0.66 & -0.64 & -0.47 & -0.48 & -0.46 & -0.62 \\
\hline LV-VIX & -0.63 & -0.59 & -0.46 & -0.47 & -0.45 & -0.56 \\
\hline
\end{tabular}

TABLE 5 - Optimal portfolio allocations for global European investors

This table reports the optimal compositions and performances of the portfolios that minimize the $95 \%$ modified CVaR for MSCI EMU index and long volatility strategies (VIX and VSTOXX) over the period February 1999December 2010.

\begin{tabular}{c|c|c}
\hline & MSCI-EMU + LV-VSTOXX & MSCI-EMU + LV-VIX \\
\hline Ann. Geo. Mean & $\mathbf{5 . 9 4 \%}$ & $\mathbf{4 . 7 2 \%}$ \\
Ann. Std. Dev. & $11.55 \%$ & $10.58 \%$ \\
Max Monthly Loss & $-8.61 \%$ & $-5.85 \%$ \\
Max Monthly Gain & $15.45 \%$ & $13.96 \%$ \\
Max Drawdown* & $-11.19 \%$ & $-25.72 \%$ \\
Skewness & 1.30 & 0.67 \\
Kurtosis & 8.10 & 4.72 \\
Mod. CVaR (95\%) & $\mathbf{4 . 9 7 \%}$ & $\mathbf{5 . 1 6 \%}$ \\
\hline \hline MSCI-EMU & $\mathbf{5 4 . 5 3 \%}$ & $\mathbf{5 7 . 2 3 \%}$ \\
LV-VSTOXX & $\mathbf{4 5 . 4 7 \%}$ & - \\
LV-VIX & - & $\mathbf{4 2 . 7 7 \%}$ \\
\hline
\end{tabular}

* The maximum drawdown is defined as the largest drop of a given asset within the sample period. 
TABLE 6 - Optimal portfolio allocations for specific European country investors with VSTOXX This table reports the optimal compositions and performances of the portfolios that minimize the $95 \%$ modified CVaR for MSCI country indices and VSTOXX over the period February 1999-December 2010.

\begin{tabular}{c|c|c|c|c|c|c}
\hline & $\begin{array}{c}\text { MSCI France } \\
\text { +LV-VSTOXX }\end{array}$ & $\begin{array}{c}\text { MSCI Germany } \\
\text { +LV-VSTOXX }\end{array}$ & $\begin{array}{c}\text { MSCI Portugal } \\
\text { +LV-VSTOXX }\end{array}$ & $\begin{array}{c}\text { MSCI Ireland } \\
\text { +LV-VSTOXX }\end{array}$ & $\begin{array}{c}\text { MSCI Greece } \\
\text { +LV-VSTOXX }\end{array}$ & $\begin{array}{c}\text { MSCI Spain } \\
\text { +LV-VSTOXX }\end{array}$ \\
\hline Ann. Geo. Mean & $\mathbf{5 . 9 4 \%}$ & $\mathbf{7 . 1 0 \%}$ & $\mathbf{3 . 8 9 \%}$ & $\mathbf{0 . 0 1 \%}$ & $\mathbf{1 . 0 7 \%}$ \\
Ann. Std. Dev. & $11.55 \%$ & $14.34 \%$ & $13.22 \%$ & $18.59 \%$ & $19.19 \%$ \\
Max Monthly Loss & $-8.61 \%$ & $-11.13 \%$ & $-7.97 \%$ & $-13.05 \%$ & $-12.75 \%$ \\
Max Monthly Gain & $15.45 \%$ & $19.01 \%$ & $13.58 \%$ & $21.80 \%$ & $15.24 \%$ \\
Max Drawdown* & $-11.20 \%$ & $-17.00 \%$ & $-20.43 \%$ & $-40.46 \%$ & $-43.58 \%$ \\
Skewness & 1.30 & 1.36 & 0.42 & 0.86 & 0.05 & $-15.96 \%$ \\
Kurtosis & 8.10 & 8.53 & 3.74 & 5.60 & 0.60 & 2.58 \\
Mod. CVaR (95\%) & $\mathbf{4 . 9 7 \%}$ & $\mathbf{6 . 1 7 \%}$ & $\mathbf{6 . 8 4 \%}$ & $\mathbf{9 . 3 9 \%}$ & $\mathbf{1 0 . 7 1 \%}$ & $\mathbf{5 . 8 3 \%}$ \\
\hline \hline Equity Index & $\mathbf{5 4 . 5 3 \%}$ & $\mathbf{5 4 . 8 4 \%}$ & $\mathbf{6 0 . 7 6 \%}$ & $\mathbf{5 1 . 4 6 \%}$ & $\mathbf{6 3 . 6 0 \%}$ & $\mathbf{5 7 . 9 9 \%}$ \\
LV-VSTOXX & $\mathbf{4 5 . 4 7 \%}$ & $\mathbf{4 5 . 1 6 \%}$ & $\mathbf{3 9 . 2 4 \%}$ & $\mathbf{4 8 . 5 4 \%}$ & $\mathbf{3 6 . 4 0 \%}$ & $\mathbf{4 2 . 0 1 \%}$ \\
\hline
\end{tabular}

* The maximum drawdown is defined as the largest drop of a given asset within the sample period.

TABLE 7 - Optimal portfolio allocations for specific European country investors with VIX This table reports the optimal compositions and performances of the portfolios that minimize the $95 \%$ modified CVaR for MSCI country indices and VIX over the period February 1999-December 2010.

\begin{tabular}{c|c|c|c|c|c|c}
\hline & $\begin{array}{c}\text { MSCI France } \\
\text { +LV-VIX }\end{array}$ & $\begin{array}{c}\text { MSCI Germany } \\
\text { +LV-VIX }\end{array}$ & $\begin{array}{c}\text { MSCI Portugal } \\
\text { +LV-VIX }\end{array}$ & $\begin{array}{c}\text { MSCI Ireland } \\
\text { +LV-VIX }\end{array}$ & $\begin{array}{c}\text { MSCI Greece } \\
\text { +LV-VIX }\end{array}$ & $\begin{array}{c}\text { MSCI Spain } \\
\text { +LV-VIX }\end{array}$ \\
\hline Ann Geo. Mean & $\mathbf{4 . 7 2 \%}$ & $\mathbf{5 . 7 6 \%}$ & $\mathbf{3 . 0 7 \%}$ & $\mathbf{- 1 . 9 3 \%}$ & $\mathbf{- 0 . 0 4 \%}$ & $\mathbf{6 . 2 2 \%}$ \\
Ann Std. Dev. & $10.60 \%$ & $13.77 \%$ & $12.88 \%$ & $15.83 \%$ & $18.60 \%$ & $12.41 \%$ \\
Max Monthly Loss & $-5.85 \%$ & $-7.03 \%$ & $-7.81 \%$ & $-12.46 \%$ & $-13.32 \%$ & $-8.95 \%$ \\
Max monthly Gain & $13.94 \%$ & $20.96 \%$ & $12.59 \%$ & $20.41 \%$ & $15.96 \%$ & $15.70 \%$ \\
Max Drawdown* & $-25.70 \%$ & $-29.36 \%$ & $-24.20 \%$ & $-45.54 \%$ & $-47.49 \%$ & $-20.86 \%$ \\
Skewness & 0.67 & 1.02 & 0.16 & 0.47 & -0.08 & 0.49 \\
Kurtosis & 4.71 & 6.72 & 3.06 & 5.06 & 3.01 & 4.54 \\
Mod. CVaR(95\%) & $\mathbf{5 . 1 6 \%}$ & $\mathbf{6 . 5 1 \%}$ & $\mathbf{7 . 0 4 \%}$ & $\mathbf{9 . 4 3 \%}$ & $\mathbf{1 1 . 2 3 \%}$ & $\mathbf{6 . 4 3 \%}$ \\
\hline \hline Equity Index & $\mathbf{5 7 . 2 3 \%}$ & $\mathbf{5 5 . 5 1 \%}$ & $\mathbf{5 8 . 5 8 \%}$ & $\mathbf{5 7 . 1 9 \%}$ & $\mathbf{6 2 . 8 1 \%}$ & $\mathbf{5 4 . 9 4 \%}$ \\
LV-VIX & $\mathbf{4 2 . 7 7 \%}$ & $\mathbf{4 4 . 4 9 \%}$ & $\mathbf{4 1 . 4 2 \%}$ & $\mathbf{4 2 . 8 1 \%}$ & $\mathbf{3 7 . 1 9 \%}$ & $\mathbf{4 5 . 0 6 \%}$ \\
\hline
\end{tabular}

* The maximum drawdown is defined as the largest drop of a given asset within the sample period. 